\title{
Does model complexity improve pricing accuracy? The case of CoCos
}

\author{
Christian Koziol $^{1}$ (D) Sebastian Weitz ${ }^{1}$
}

Accepted: 13 April 2021 / Published online: 12 May 2021

(C) The Author(s) 2021

\begin{abstract}
In this study, we analyze whether model complexity improves accuracy of CoCo pricing models. We compare the out-of-sample pricing ability of four models using a broad dataset that contains all CoCos which were issued between January 1, 2013 and May 31, 2016 in euros. The regarded models include the standard model from De Spiegeleer and Schoutens (J Deriv 20:27-36, 2012), a modified version enriched by credit risk, an extended model that accounts for the effective lifetime of the CoCo, and a trading model, solely based on historic market prices but no pricing theory at all. For a normal market environment, the simple trading model provides a higher pricing accuracy than the theory-based models. Under distress, however, a theory-based model with a sufficiently high complexity is required.
\end{abstract}

Keywords Contingent convertible bond · CoCo bond · CoCo pricing ·

Continuous-time derivatives pricing $\cdot$ Model complexity $\cdot$ Test of pricing models

Mathematics Subject Classification G12 - G13

\section{Introduction}

Contingent convertible bonds (CoCos) are a modern financing instrument for banks intended to enhance stability. This favorable characteristic comes at the expense that several features make CoCos highly complex and their accurate pricing a complicated task for investors. First, when a bank violates a pre-specified regulatory condition, the CoCo is triggered and the initial coupon bond is converted into stocks (or accordingly suffers a write-down). This special term requires regulatory and/or accounting

\footnotetext{
$\bowtie$ Christian Koziol

christian.koziol@uni-tuebingen.de

Sebastian Weitz

sebastian.weitz@uni-tuebingen.de

1 Department of Finance, University of Tuebingen, Nauklerstr. 47, 72074 Tuebingen, Germany
} 
knowledge and is therefore much more challenging than a plain vanilla option exercise. Second, CoCos are perpetual and callable by the issuer but usually have an initial blocking period. Hence, the effective lifetime is not known in advance and depends on the call strategy carried out by the issuer. Third, CoCos are influenced by multiple sources of risk: the stock price, the interest rate, and the issuer's solvency.

In an empirical exercise considering only three first generation CoCo issuances, Wilkens and Bethke (2014) assess the pricing performance of different types of pricing models. In particular, they regard models from the following three classes: structural models such as Pennacchi (2011), Buergi (2013) and Brigo et al. (2015) in which the trigger event is determined by modelling the balance sheet, credit derivatives models such as Serjantov (2011), De Spiegeleer and Schoutens (2014), Cheridito and Xu (2015) and Turfus and Shubert (2017) which address the additional yield compensation for the exposure to trigger risk, as well as equity derivatives models like De Spiegeleer and Schoutens (2012) and Corcuera et al. (2013) which focus on the replication of the CoCo value using equity derivatives. The findings of Wilkens and Bethke (2014) favor the use of the equity derivatives model of De Spiegeleer and Schoutens (2012) compared to both balance sheet and credit risk models from the other two classes.

The objective of our study is to analyze whether models that are more complex, in fact, provide a higher pricing accuracy. Higher complexity does not automatically guarantee improved pricing accuracy due to the well-known problem of overfitting. Since the pricing of CoCos is especially challenging, we focus on this type of securities for our analysis. In contrast to Wilkens and Bethke (2014), we use a broad dataset comprised of 27 CoCos all issued between January 1, 2013 and May 31, 2016 denominated in euros. This broad dataset enables us to figure out to what extend a more sophisticated model can explain CoCo values in a better way.

For this purpose, we consider four models: As our first model, we refer to the standard approach from De Spiegeleer and Schoutens (2012). The second model is a modified version of the standard approach that also allows for credit risk in a closed-form pricing formula. The closed-form pricing formulae of the first two models, however, are at the expense of a simplifying maturity structure disregarding the effective lifetime of the CoCo. Thus, we need to develop an extended approach as our third model that reasonably accounts for the maturity structure by modelling the effective trigger date, the perpetual lifetime, the initial blocking period, and a potential call by the issuer. Finally, our fourth model is a market-based approach which solely bases on observable market prices but no pricing theory at all.

Our study provides four major results: first, information on credit risk stabilizes the price prediction and should therefore be included in a CoCo pricing model. Second, the extended model, which endogenously captures the lifetime of the CoCo, results in a higher out-of-sample accuracy than the other theory-based models in closed-form with and without credit risk. Therefore, the consideration of the effective lifetime is highly recommended. Third, in comparison to the market-based approach, the extended model is no longer dominant but a simple model without pricing theory can be justified on average. Fourth, in distressed markets, the market-based approach is comparably more exposed to severe mispricing than the more complex extended model which remains comparably robust in this situations. This finding leads to the conclusion that in an ordinary market environment a simple market-based approach might be sufficient for 
accurate CoCo pricing. Under distress, however, a more complex model is required which considers the CoCo's product features. Thus, we need to distinguish between pricing accuracy on average and robustness in distressed markets.

The remainder of our study is organized as follows. In Sect. 2, we introduce the pricing models. Section 3 contains the empirical analysis in which we present the data, the calibration procedure and the evaluation of the out-of-sample pricing performance of the models. In Sect. 4, we focus on distressed market phases and evaluate the models pricing performance. Section 5 concludes. Technical derivations are in the "Appendix".

\section{Pricing models}

In order to analyze CoCo models in terms of their pricing abilities, we particularly select the following four models:

1. The standard model (SM) introduced by De Spiegeleer and Schoutens (2012).

2. The default risk model (DM), an enhancement of the De Spiegeleer and Schoutens (2012) pricing model to include credit risk.

3. The extended model (EM) which additionally captures the corresponding lifetime of the CoCo in form of the effective trigger date, the perpetual lifetime, the initial blocking period, and a potential call by the issuer.

4. The trading model (TM) which only obtains the CoCo's exposures for the risk factors from historic market prices.

The standard model (SM) provides a widely established closed-form solution for the CoCo price in a Black-Scholes framework (see Black and Scholes 1973). In the numerical CoCo exercise carried out by Wilkens and Bethke (2014), this model exhibited the most promising results. Since credit risk is apparently a further major source of risk, we secondly modify the standard model into a default risk model (DM) by incorporating credit risk. Our third pricing theory-based model, the extended model (EM), additionally allows for an appropriate lifetime by accounting for the correct trigger date, the perpetual lifetime with an initial blocking period, and the issuer's call right. In this case, a closed-form solution is no longer possible. The fourth version, the trading model (TM), ignores any potential pricing theory-based formulae for the CoCo and only relies on observable market relations. We can interpret this trading model as a case of a market participant who empirically determines hedge ratios without knowledge about CoCo pricing theory.

\subsection{Standard model}

The standard model proposed by De Spiegeleer and Schoutens (2012) resorts to a Black-Scholes world, in which the issuer's stock price is governed by

$$
d S_{t}=(r-q) S_{t} d t+\sigma S_{t} d z_{t},
$$


where $r$ denotes the risk-free rate, $q$ the dividend yield and $\sigma$ the instantaneous standard deviation of the issuer's stock returns. All risk comes from $d z_{t}$ which is the increment of a standard Wiener process under the risk-neutral measure. We assume $r, q$ and $\sigma$ to be constant.

For the beginning, we follow De Spiegeleer and Schoutens (2012) by only focusing on conversion CoCos. Later on in this section we will modify their approach such that it can also be applied to value write-down CoCos. In principle, unless specified, the term CoCo refers to both types.

De Spiegeleer and Schoutens (2012) value a conversion CoCo by replicating its payoff at a fixed maturity date $T$ that is given by the end of the call blocking period. Thus, in the model the CoCo no longer exists after this date. The restrictive maturity assumption enables them to obtain tractable pricing formulae using the following three traded securities as building blocks: First, a coupon bond accounts for the coupon distributions and the notional amount. Second, a knock-in forward that accounts for the trigger event by a conversion into equity, and third, a binary down-and-in option that corrects for lost payments once the CoCo is triggered.

The first building block $B_{t}^{(1)}(T)$ is represented by a coupon bond, that pays $K$ coupons $C$ at dates $\tau_{1}, \tau_{2}, \ldots, \tau_{K}$ with notional amount $N$ until it matures at the end of the blocking period at time $T$. The value of this first building block $B_{t}^{(1)}(T)$ is therefore given by:

$$
B_{t}^{(1)}(T)=\sum_{i=1}^{K} C e^{-r \cdot\left(\tau_{i}-t\right)}+N e^{-r \cdot(T-t)}
$$

Once the issuer's stock price $S$ hits a trigger threshold $\underline{S}$ from above, the trigger event occurs and the CoCo gets converted. Here, the investor receives $n$ stocks but loses all coupon payments and the notional amount $N$. De Spiegeleer and Schoutens (2012) incorporate this by using a knock-in forward as a second building block $B_{t}^{(2)}(T, q)$, that ensures delivery of $n$ stocks at a price $N$ at maturity $T$ when the trigger threshold $\underline{S}$ was hit until maturity. The value of a knock-in forward $B_{t}^{(2)}(T, q)$ reads (see Rubinstein and Reiner 1991a) ${ }^{1}$

$$
\begin{gathered}
B_{t}^{(2)}(T, q)=n \cdot S_{t} e^{-q \cdot(T-t)}\left[\left(\frac{\underline{S}}{S_{t}}\right)^{2 \eta} \mathcal{N}\left(y_{1}\right)+\mathcal{N}\left(-x_{1}\right)\right] \\
-N e^{-r \cdot(T-t)}\left[\left(\frac{\underline{S}}{S_{t}}\right)^{2 \cdot(\eta-1)} \mathcal{N}\left(y_{2}\right)+\mathcal{N}\left(-x_{2}\right)\right]
\end{gathered}
$$

with

$$
y_{1}=\frac{\ln \left(\frac{\underline{S}}{S_{t}}\right)+\eta \cdot \sigma^{2} \cdot(T-t)}{\sigma \sqrt{T-t}} \quad x_{1}=\frac{\ln \left(\frac{S_{t}}{\underline{S}}\right)+\eta \cdot \sigma^{2} \cdot(T-t)}{\sigma \sqrt{T-t}}
$$

\footnotetext{
1 Please note, we specifically refer to the dividend yield, since it will play a certain role when considering default risk in the subsequent section.
} 


$$
y_{2}=y_{1}-\sigma \sqrt{T-t} \quad x_{2}=x_{1}-\sigma \sqrt{T-t} \quad \eta=\frac{r-q+\frac{1}{2} \sigma^{2}}{\sigma^{2}}
$$

To correct for the vanishing coupons once the CoCo was triggered, De Spiegeleer and Schoutens (2012) consider a binary down-and-in option with barrier $\underline{S}$ paying one monetary unit at expiration $\tau$ if the CoCo was triggered and zero otherwise. The value of this third building block $B_{t}^{(3)}(\tau, q)$ can be obtained by (see Rubinstein and Reiner 1991b):

$$
B_{t}^{(3)}(\tau, q)=e^{-r \cdot(\tau-t)}\left[\mathcal{N}\left(-x_{2, \tau}\right)+\left(\frac{\underline{S}}{S_{t}}\right)^{2 \cdot(\eta-1)} \mathcal{N}\left(y_{2, \tau}\right)\right]
$$

with

$y_{2, \tau}=\frac{\ln \left(\frac{\underline{S}}{S_{t}}\right)+(\eta-1) \cdot \sigma^{2} \cdot(\tau-t)}{\sigma \sqrt{\tau-t}} \quad x_{2, \tau}=\frac{\ln \left(\frac{S_{t}}{\underline{S}}\right)+(\eta-1) \cdot \sigma^{2} \cdot(\tau-t)}{\sigma \sqrt{\tau-t}}$

De Spiegeleer and Schoutens (2012) replicate a CoCo by a portfolio consisting of long positions in a coupon bond and a knock-in forward as well as $K$ short positions in $C$ binary down-and-in options, each expiring at the corresponding coupon payment dates.

In summary, if the stock price remains above the trigger barrier $\underline{S}$, the CoCo pays periodic coupons and the investor receives the notional amount at the end of the blocking period at time $T$. If the CoCo is triggered, the knock-in forward comes into play. Here, the investor receives a package of $n$ forwards on the issuers stock at a total price equal to the notional amount $N$ and the remaining coupons are lost due to the binary down-and-in options.

We now turn to the case of write-down CoCos. This step is necessary because write-down CoCos make up approximately half of our dataset, which is in line with the global distribution by type (see Avdjiev et al. 2017). We modify the framework of De Spiegeleer and Schoutens (2012) by replacing the knock-in forward with a short position in $\alpha N$ binary down-and-in options that expire at $T$ and write down the notional amount accordingly by the write-down fraction $\alpha$. Furthermore, the payments of the binary down-and-in options that correct for the vanishing coupons are also written down, so that a short position of $\alpha C$ binary down-and-in options for each of the $K$ coupon payment dates is required. Again, once the CoCo is triggered, it will turn into a coupon bond maturing at $T$ that received a haircut of $\alpha$ percent. In absence of a trigger event, the CoCo is due at the end of the blocking period.

Combining the buildings blocks $B_{t}^{(1)}(T), B_{t}^{(2)}(T, q)$, and $B_{t}^{(3)}(\tau, q)$, today's price $P_{t}^{S M}(T, q)$ for both CoCo types, conversion and write-down, maturing at time $T$ in the standard model (SM) is given by: 


$$
P_{t}^{S M}(T, q)=\left\{\begin{array}{lc}
B_{t}^{(1)}(T)+n \cdot B_{t}^{(2)}(T, q)-C \cdot \sum_{i=1}^{K} B_{t}^{(3)}\left(\tau_{i}, q\right) & \begin{array}{cc}
\text { Conversion } \\
\text { CoCo }
\end{array} \\
B_{t}^{(1)}(T)-\alpha N \cdot B_{t}^{(3)}(T, q)-\alpha C \cdot \sum_{i=1}^{K} B_{t}^{(3)}\left(\tau_{i}, q\right), & \text { Write-down } \\
\text { CoCo }
\end{array}\right.
$$

The advantage of this model is its tractability due to the closed-form solution. However, this benefit come from very restrictive assumptions: first, considering only a fixed maturity, the model of De Spiegeleer and Schoutens (2012) does not account for the CoCo's effective lifetime. Since CoCos must be perpetual in order to be eligible as AT1 capital and are callable by the issuer after an initial blocking period, the very restrictive assumption of a fixed maturity by De Spiegeleer and Schoutens (2012) distorts the interest rate sensitivity of the CoCo. Second, AT1 CoCos are deeply subordinated securities that are exposed to credit risk. This additional source of risk cannot be captured in this simple framework omitting credit risk.

\subsection{Default risk model}

In the next step, we maintain the restrictive maturity assumption of De Spiegeleer and Schoutens (2012), but allow for a sudden default of the issuer by incorporating credit risk into the model. Therefore, we specify the issuer's stock price and solvency simultaneously and assume the latter to follow a Poisson process $J_{t}$ with a constant jump intensity $\lambda$. Intuitively speaking, $J_{t}$ is initially zero at time $t=0$ and jumps to $J_{t}=1$ at the first jump date which is assumed to be independent of the stock price state. As a consequence, the issuer defaults at the first jump date, the stock price drops to zero and the CoCo expires worthless, too.

Since we are in a world free of arbitrage, there exists an equivalent martingale measure under which the stock price process discounted by the risk-free rate is a martingale. To ensure this property, we incorporate the default intensity $\lambda$ into the drift of our stochastic differential equation for the issuer's stock price:

$$
d S_{t}=(r-q+\lambda) S_{t} d t+\sigma S_{t} d z_{t}-S_{t} d J_{t}
$$

with $q^{\prime}=q-\lambda$. As long as the issuer survives, the CoCo behaves analogously to the standard model with modified dividend yield $q^{\prime}$ and we can value the CoCo using the standard model formula given in Eq. (5). Though, we have to account for the changed drift in Eq. (6) by using the building blocks $B_{t}^{(2)}\left(T, q^{\prime}\right)$ and $B_{t}^{(3)}\left(\tau, q^{\prime}\right)$. In case of default, the CoCo expires worthless.

Following risk-neutral valuation, we obtain the value of the CoCo with credit risk by weighting the pricing formula of the standard model $P_{t}^{S M}\left(T, q^{\prime}\right)$ with the riskneutral probability of default $p d_{t, T}$ while accounting for the changed drift using $q^{\prime}$. Therefore, the pricing formula $P_{t}^{D M}\left(T, q^{\prime}\right)$ for the default risk model (DM) follows:

$$
P_{t}^{D M}\left(T, q^{\prime}\right)=\left(1-p d_{t, T}\right) \cdot P_{t}^{S M}\left(T, q^{\prime}\right)
$$


with the risk-neutral probability of default until time $T$

$$
p d_{t, T}=\int_{t}^{T} \lambda e^{-\lambda \cdot(s-t)} d s=1-e^{-\lambda \cdot(T-t)} .
$$

\subsection{Extended model}

The aim of the extended model is to capture the maturity structure more accurately. Our improvements relative to the standard model (SM) and the default risk model (DM) are threefold: first, we model the correct trigger time $\tau_{C}$, thereby accounting for potential dividend payments appropriately. Second, we no longer use a fixed maturity but model the $\mathrm{CoCo}$ as a perpetual security. This is a crucial requirement from the regulator in order to be eligible as AT1 capital. Third, the issuer can call the CoCo at the call price $\bar{P}$ after an initial blocking period of $T$ years. These three extensions are in line with major contractual details of traded AT1 CoCos and allow the effective lifetime to be appropriately considered. It is expected that these extensions will improve the pricing performance relative to the standard model in Sect. 2.1 and the default risk model in Sect. 2.2.

Figure 1 illustrates the consequences for the CoCo depending on different stock price evolutions within the extended model framework. Looking at path $A^{(1)}$, the survivorship case, the CoCo survives the initial blocking period because the issuer's stock price reaches neither the call $\bar{S}$ nor the trigger barrier $\underline{S}$. Hence, the CoCo still exists after the blocking period ends at $T$ and coupon rate $\bar{c}$ is still paid. Path $A^{(2)}$ shows the call-scenario, in which the stock price ends up at or above the call barrier $\bar{S}$ at the end of the initial blocking period. Thus, as soon as permissible i.e., at time $T$, the issuer calls the CoCo at the call price $\bar{P}$. In the trigger scenario $B$, the trigger barrier $\underline{S}$ is hit at time $\tau_{C}$. Consequently, the CoCo is triggered. Last, path $\mathrm{C}$ shows the default scenario in which the stock price jumps to zero at $\tau_{D}$ and the CoCo becomes worthless.

\section{CoCo after the blocking period}

For pricing purposes, it is helpful to structure the CoCo framework into two periods: within and after the initial blocking period. We begin with a CoCo after the blocking period, which is illustrated to the right of the vertical line at $T$ in Fig. 1.

Analogously to Sects. 2.1 and 2.2, we replicate the payout structure of the CoCo using a portfolio out of building blocks that are given in the form of perpetual state prices $\underline{C}_{t}$ and $\bar{C}_{t}$ written on the issuer's stock price process.

Having a trigger event in mind, we derive a state price $\underline{C}_{t}$ that pays one unit if the stock price hits a lower boundary $\underline{S}$ and ceases to exist if the stock price hits an upper boundary $\bar{S}$ or defaults. Within the boundaries the claim continues without any payoffs. The value of this state price $\underline{C}_{t}$ is given by

$$
\underline{C}_{t}=\frac{1}{\underline{S}^{\gamma_{1}}-\underline{S}^{\gamma_{2}} \bar{S}^{\gamma_{1}-\gamma_{2}}} S_{t}^{\gamma_{1}}+\frac{1}{\underline{S}^{\gamma_{2}}-\underline{S}^{\gamma_{1}} \bar{S}^{\gamma_{2}-\gamma_{1}}} S_{t}^{\gamma_{2}}
$$




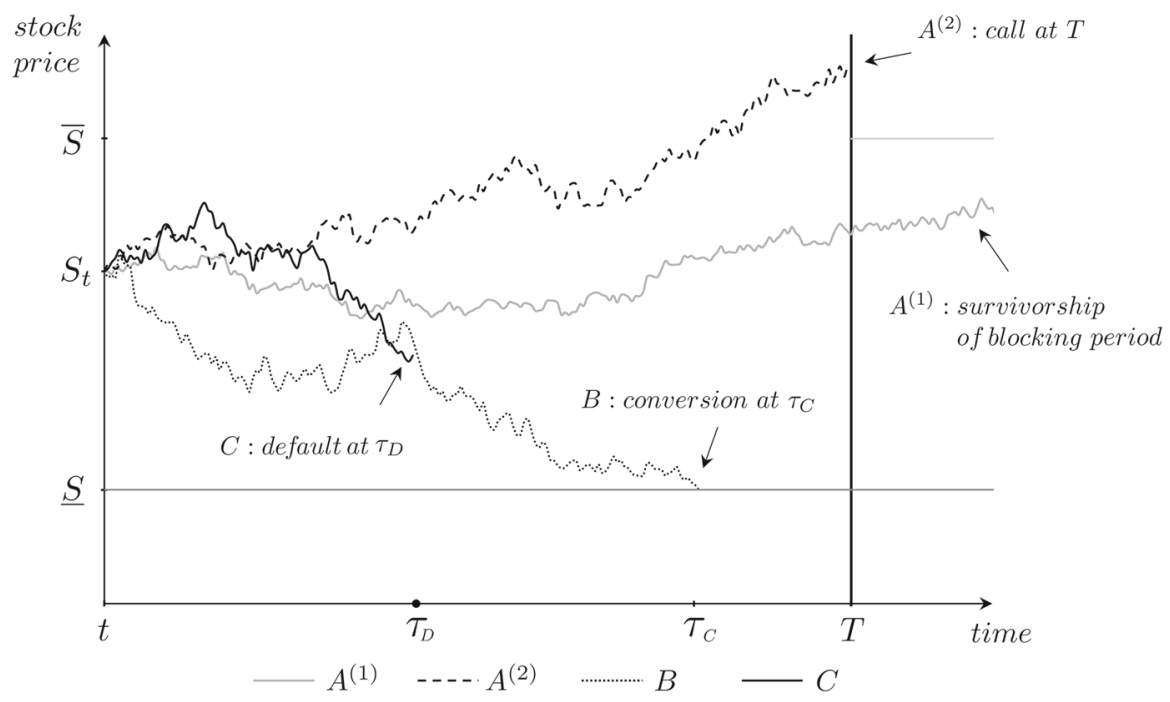

Fig. 1 Extended model framework.

This graph shows the four possible stock price evolutions across time and the corresponding consequences for the CoCo within the extended model framework. The horizontal lines at $\underline{S}$ and $\bar{S}$ indicate the trigger and call barriers. The vertical line at $T$ indicates the end of the initial blocking period at time T. Path $A^{(1)}$ shows a situation in which the CoCo is still alive after the blocking period. In $A^{(2)}$ the issuer calls the CoCo at $T$ since the stock price $S$ increased above $\bar{S}$. A trigger scenario is shown in path $B$ in which the stock price hits $\underline{S}$ at time $\tau_{C}$. In path $C$, the issuer defaults at time $\tau_{D}$ and the stock price jumps to zero

with constant exponents

$$
\gamma_{1}=\frac{1}{\sigma^{2}}\left[q^{\prime}-r+\frac{1}{2} \sigma^{2}-\sqrt{2(r+\lambda) \sigma^{2}+\frac{1}{4}\left(2\left(q^{\prime}-r\right)+\sigma^{2}\right)^{2}}\right]
$$

and

$$
\gamma_{2}=\frac{1}{\sigma^{2}}\left[q^{\prime}-r+\frac{1}{2} \sigma^{2}+\sqrt{2(r+\lambda) \sigma^{2}+\frac{1}{4}\left(2\left(q^{\prime}-r\right)+\sigma^{2}\right)^{2}}\right]
$$

The derivation of Eq. (9) is provided in the "Appendix".

Similarly, the value of a state price $\bar{C}_{t}$ that pays one unit at $\bar{S}$ and expires worthless at $\underline{S}$ or at default reads:

$$
\bar{C}_{t}=\frac{1}{\bar{S}^{\gamma_{1}}-\bar{S}^{\gamma_{1}} \underline{S}^{\gamma_{1}-\gamma_{2}}} S_{t}^{\gamma_{1}}+\frac{1}{\bar{S}^{\gamma_{2}}-\bar{S}^{\gamma_{1}} \underline{S}^{\gamma_{2}-\gamma_{1}}} S_{t}^{\gamma_{2}}
$$

Given the stock price is within the barriers, the CoCo has the character of a perpetual coupon bond that is subject to default risk. In this case, the value is given by $\frac{c}{r+\lambda}$. Once the stock price hits $\underline{S}$, the CoCo is triggered and takes on the value $\underline{P}$, which is either the value of the share package $n \cdot \underline{S}$ or the value of the perpetual coupon bond after the write-down $(1-\alpha) \cdot \frac{c}{r+\lambda}$. Likewise, if the stock price reaches the call barrier 
$\bar{S}$, the issuer calls the CoCo at the call price $\bar{P}$. In addition, if the stock price after the blocking period at time $T$ is above $\bar{S}$, the issuer calls immediately at the call price $\bar{P}$. Thus, in the extended model (EM) we obtain the price $P_{t}^{E M, P}$ of a CoCo after the initial blocking period at time $t>T$ :

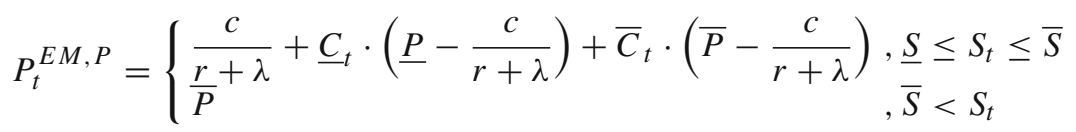

The CoCo value $P_{t}^{E M, P}$ can be interpreted as a credit risky bond $\frac{c}{r+\lambda}$ with extra payment $\underline{P}-\frac{c}{r+\lambda}$ in case of a trigger event or $\bar{P}-\frac{c}{r+\lambda}$ in case of a call. These potential payments are weighted in Eq. (11) with the corresponding state prices.

In the case of a trigger event, the CoCo takes on the trigger value $\underline{P}$

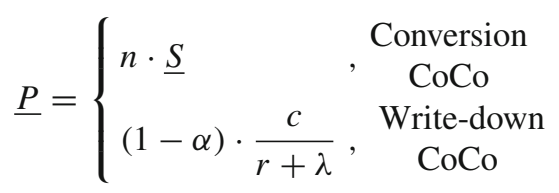

While the conditions under which the CoCo is triggered are contractually fixed and thus exogenously given by the trigger barrier $\underline{S}$, the call barrier $\bar{S}$ follows endogenously from the issuer's optimal behavior. We assume a call strategy that follows the ideas of Brennan and Schwartz (1977) and Ingersoll (1977), in which the issuer calls the CoCo so that the value is minimized. We can determine the optimal call barrier using the smooth-pasting condition (see Sarkar 2003):

$$
\left.\frac{\partial P^{E M, P}}{\partial S}\right|_{S=\bar{S}^{*}}=0
$$

The left side of Eq. (13) corresponds to the derivative of the CoCo pricing function in Eq. (11) for the stock price $S$ evaluated at the call barrier $\bar{S}$ :

$$
\frac{\left(\bar{P}-\frac{c}{r+\lambda}\right)\left(\gamma_{1} \underline{S}^{\gamma_{2}} \bar{S}^{\gamma_{1}}-\gamma_{2} \underline{S}^{\gamma_{1}} \bar{S}^{\gamma_{2}}\right)-\left(\underline{P}-\frac{c}{r+\lambda}\right)\left(\gamma_{1}-\gamma_{2}\right) \bar{S}^{\gamma_{1}+\gamma_{2}}}{\bar{S}\left(\underline{S}^{\gamma_{2}} \bar{S}^{\gamma_{1}}-\underline{S}^{\gamma_{1}} \bar{S}^{\gamma_{2}}\right)}=0
$$

The optimal call barrier $\bar{S}^{*}$ solves Eq. (14) and must be obtained numerically.

\section{CoCo within the blocking period}

In a next step, we consider a CoCo within the blocking period, which is graphically illustrated in Fig. 1 by the area to the left of $T$. Three different scenarios exist within this period: The CoCo survives without being trigger or defaulted (A: $T<\tau_{C} \wedge T<\tau_{D}$ ). The CoCo is triggered before the issuer defaults (B: $\tau_{C}<T \wedge \tau_{C}<\tau_{D}$ ). The issuer 
defaults before the CoCo is triggered within the blocking period $\left(\mathrm{C}: \tau_{D}<T \wedge \tau_{D}<\right.$ $\left.\tau_{C}\right)$.

In our extended model (EM), we value $P_{t}^{E M}$ the CoCo within the blocking period as the expectation of the discounted mutually exclusive payoff-scenarios under the risk-neutral measure as

$$
P_{t}^{E M}=E_{t}\left[A \cdot \mathbb{1}_{\left\{T<\tau_{C} \wedge T<\tau_{D}\right\}}+B \cdot \mathbb{1}_{\left\{\tau_{C}<T \wedge \tau_{C}<\tau_{D}\right\}}+C \cdot \mathbb{1}_{\left\{\tau_{D}<T \wedge \tau_{D}<\tau_{C}\right\}}\right]
$$

$A, B$ and $C$ stand for the value of the CoCo depending on the respective stock price scenario during the blocking period (BP) which is indicated by the indicator function $\mathbb{1}_{\{.\}}$.

$$
\begin{array}{ll}
A=P_{T}^{E M, P} \cdot e^{-r \cdot(T-t)}+\frac{c}{r} \cdot\left(1-e^{-r \cdot(T-t)}\right) & \text { Survivorship of BP } \\
B=\frac{P}{c} \cdot e^{-r \cdot\left(\tau_{C}-t\right)}+\frac{c}{r} \cdot\left(1-e^{-r \cdot\left(\tau_{C}-t\right)}\right) & \text { Trigger within BP } \\
C=\frac{c}{r} \cdot\left(1-e^{-r \cdot\left(\tau_{D}-t\right)}\right) & \text { Default within BP }
\end{array}
$$

In scenario A the CoCo survives the initial blocking period. At the end of the blocking period, the stock price is either at/above the call barrier $\bar{S}$ and the issuer calls the CoCo immediately (path $A^{(1)}$ in Fig. 1) or it is between the call and trigger barriers (path $A^{(2)}$ ) and the CoCo becomes callable. In both situations, the CoCo pays coupons with present value of $\frac{c}{r} \cdot\left(1-e^{-r \cdot(T-t)}\right)$ throughout the blocking period. If triggered within the blocking period at time $\tau_{C}$ (scenario B), the CoCo equals the value of a share package (coupon bond after write-down). Furthermore, the CoCo pays coupons until $\tau_{C}$ with present value of $\frac{c}{r} \cdot\left(1-e^{-r \cdot\left(\tau_{C}-t\right)}\right)$. In scenario $\mathrm{C}$, the issuer defaults before a trigger event and the CoCo expires worthless. The CoCo, however, pays coupons until the default event occurs at $\tau_{D}$. This amounts to $\frac{c}{r} \cdot\left(1-e^{-r \cdot\left(\tau_{D}-t\right)}\right)$.

Accounting for the appropriate trigger date, the perpetual lifetime, the call right of the issuer, and the initial blocking period of CoCos enables us to capture the effective maturity more accurately. However, this is at the expense of a closed-form solution within the blocking period.

\subsection{Trading model}

In a last step, we use a market-based approach, the trading model, that purely focusses on observed prices and fully ignores any kind of pricing model or formulae. This serves as a benchmark for the other models.

For this purpose, we introduce an empirical approach and predict CoCo price changes using its underlying risk exposures. Due to the hybrid nature of CoCos, we consider credit, equity and interest rate risk as the crucial drivers. We set up the following linear relationship to explain CoCo price changes in the trading model (TM):

$$
\Delta P_{t}^{T M}=\beta_{S} \cdot \Delta S_{t}+\beta_{c s} \cdot \Delta c s_{t}+\beta_{r} \cdot \Delta r_{t}
$$


where $\Delta S_{t}$ is the change of the issuer's stock price, $\Delta c s_{t}$ is the change in the credit default swap spread of the issuer and $\Delta r_{t}$ is the yield change on the risk-free asset. The empirical exposures $\beta_{S}, \beta_{r}$ and $\beta_{c s}$ are estimated using time series regression.

\section{Pricing accuracy}

\subsection{Data}

Our CoCo sample consists of all AT1 CoCos issued between January 1, 2013 and May 31, 2016 denominated in euros. Table 1 provides an overview. Twenty banks issued $27 \mathrm{CoCos}$ with a total volume of 26.9 billion euros. The dataset contains 13 equity conversion and 14 write-down CoCos. The average CoCo in our sample pays a coupon of $6.88 \%$ (usually semi-annually or quarterly) and can be called after an initial blocking period of 6 years. We obtained information on the coupon size, the coupon payment frequency, the issuance date, the first call date, the CoCo type as well as the write-down fraction or the size of the share package from the offering memorandum. Although CoCos are contractually triggered by a violation of a pre-specified CET1 ratio, we we do not refer to this accounting numbers but consider an implicit trigger level $\underline{S}$ of the stock price. First, accounting numbers are known to be backward-oriented which prevents a proper indication of a CET1 ratio violation due to current changes in the issuers solvency (see Flannery 2016). Second, an unobservable and presumably time-dependent regulatory trigger, which accompanies the accounting trigger, is likely to precede indications of solvency issues in the CET1 ratio. This situation can been seen in the case of Banco Popular Español. In June 2016, the European Central Bank (ECB) classified the bank as 'failing or likely to fail' (Article 32, Bank Recovery and Resolution Directory) and enforced immediate conversion of the CoCos although the bank's CET1 ratio was above the contractual trigger threshold (see Pennacchi and Tchistyi 2018).

Market data was retrieved from Thomson Reuters Eikon on a daily basis. We use clean prices for CoCos and closing prices of the issuer's stock. The dividend yield is given by the net annualized historical dividend over the current stock price. Information on the issuers' solvency is represented by the mid-spread of five-year credit default swaps on unsecured senior debt of the issuer. We translated the daily spreads $c d s_{t}$ into instantaneous probabilities of default $\lambda_{t}$ using the following relationship

$$
\lambda_{t}=\frac{c d s_{t}}{1-\varphi}
$$

assuming a typical recovery of unsecured senior debt of the issuer $\varphi=0.4$ (see Altman et al. 2005).

The risk-free interest rates were estimated using the Svensson (1994) model for which the ECB estimates the required parameter inputs from AAA-rated European sovereign debt securities. The daily estimates are available for download on the website of the ECB. The end of the observed sample period is May 31, 2018. 
Table 1 CoCo sample

\begin{tabular}{|c|c|c|c|c|}
\hline $\mathrm{CoCo}$ & Coupon & Issue date & First call & Type \\
\hline Aareal Bank & 7.625 & Nov. 20, 2014 & Apr. 4, 2020 & WD \\
\hline ABN AMRO & 5.750 & Sep. 22, 2015 & Nov. 22, 2020 & WD \\
\hline Allied Irish Banks & 7.375 & Dec. 3, 2015 & Dec. 3, 2020 & WD \\
\hline BBVA (1) & 7.000 & Feb. 19, 2014 & Feb. 19, 2019 & $\mathrm{EC}$ \\
\hline BBVA (2) & 6.750 & Feb. 18, 2015 & Feb. 18, 2020 & $\mathrm{EC}$ \\
\hline BBVA (3) & 8.875 & Apr. 14, 2016 & Apr. 14, 2021 & EC \\
\hline Ba. Popular (1) & 11.500 & Oct. 10, 2013 & Oct. 10,2018 & $\mathrm{EC}$ \\
\hline Ba. Popular (2) & 8.250 & Feb. 12, 2015 & Apr. 10, 2020 & EC \\
\hline Bank of Ireland & 7.375 & Jun. 18, 2015 & Jun. 18, 2020 & WD \\
\hline Barclays (1) & 8.000 & Dec. 10, 2013 & Dec. 15,2020 & EC \\
\hline Barclays (2) & 6.500 & Jun. 17, 2014 & Sep. 15, 2019 & $\mathrm{EC}$ \\
\hline BNP Paribas & 6.125 & Jun. 17, 2015 & Jun. 17, 2022 & WD \\
\hline Crédit Agricole & 6.500 & Apr. 8, 2014 & Jun. 23, 2021 & WD \\
\hline Danske Bank (1) & 5.750 & Mar. 12, 2014 & Apr. 6, 2020 & WD \\
\hline Danske Bank (2) & 5.875 & Feb. 18, 2015 & Apr. 6, 2022 & WD \\
\hline Deutsche Bank & 6.000 & May 27, 2014 & Apr. 30, 2022 & WD \\
\hline HSBC (1) & 5.250 & Sep. 17, 2014 & Sep. 16, 2022 & $\mathrm{EC}$ \\
\hline HSBC (2) & 6.000 & Sep. 29, 2015 & Sep. 29, 2023 & EC \\
\hline Intesa Sanpaolo & 7.000 & Jan. 19, 2016 & Jan. 19, 2021 & WD \\
\hline KBC Group & 5.625 & Mar. 19, 2014 & Mar. 19, 2019 & WD \\
\hline Lloyds Banking & 6.375 & Apr. 1, 2014 & Jun. 27, 2020 & EC \\
\hline Permanent TSB & 8.625 & May 6, 2015 & Apr. 1, 2021 & $\mathrm{EC}$ \\
\hline Santander (1) & 6.250 & Mar. 12, 2014 & Mar. 12, 2019 & $\mathrm{EC}$ \\
\hline Santander (2) & 6.250 & Sep. 11, 2014 & Sep. 11, 2021 & EC \\
\hline Société Générale & 6.750 & Apr. 7, 2014 & Apr. 7, 2021 & WD \\
\hline UBS & 5.750 & Feb. 19, 2015 & Feb. 19, 2022 & WD \\
\hline UniCredit & 6.750 & Sep. 10, 2014 & Sep. 10, 2021 & WD \\
\hline
\end{tabular}

This table contains all CoCos issued between January 1, 2013 and May 31, 2016 denominated in euros. The sampling period for the daily market data of each CoCo starts with the issue date and ends at May 31, 2018. The only exceptions are the CoCos of Banco Popular Español for which we regard the data until June 6,2017 , the trigger date of the CoCos. The coupon size is in percentage terms. We also provide information about the type of CoCo, equity conversion (EC) or write-down (WD)

\subsection{Pricing setup}

In principle, a pricing model is represented by a function $F\left(\Omega_{t}, \Theta_{t}\right)$ that depends on observable $\Omega_{t}$ and unobservable $\Theta_{t}$ parameters. Before a model for the CoCo at a pricing date $t$ can be applied, we need to clarify of how and at which point in time we get the unobservable parameters $\Theta_{t}$ in the first place.

With respect to the latter, we think of a CoCo investor that applies the models similar to an options trader who insures her portfolio using the delta derived from the 


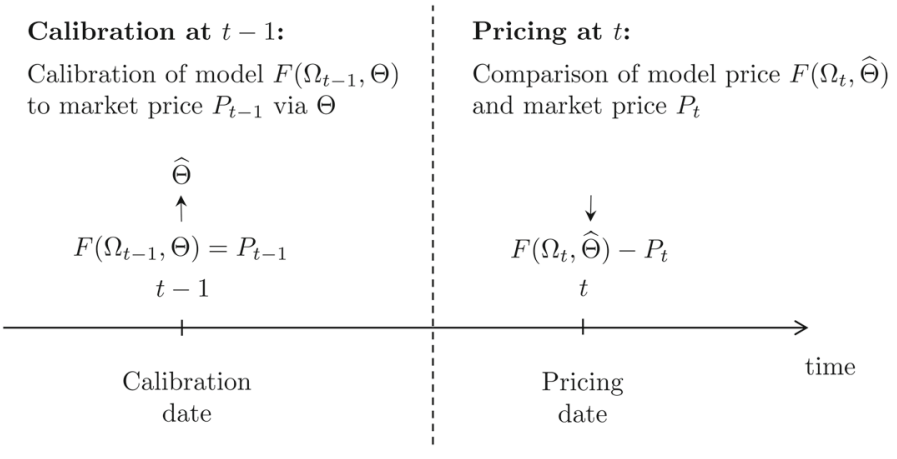

Fig. 2 Time structure of theory-based models

prominent Black and Scholes (1973) model. Since it is common that options portfolios are hedged on a daily basis, we use all information available until one day prior to the pricing date to calibrate the models, i.e., until $t-1$. Regarding the calibration procedure, we distinguish two situations depending on the model type.

In case of the theory-based models, i.e., SM, DM and EM, we proceed according to the time structure visualized in Fig. 2. In this situation, neither the trigger threshold $\underline{S}$ nor the volatility $\sigma$ of the issuer's stock price are observable. In contrast to plain vanilla option pricing, we cannot easily use the implicit volatility from observed market prices since this requires a product with comparable maturity and moneyness. With respect to moneyness, the CoCo trigger is similar in character to a deep-out-of-the-money put option. While it is already very difficult to obtain a sensible implicit volatility since such products are rarely traded, the perpetual lifetime and the call feature makes this endeavor for CoCos almost impossible.

Corcuera et al. (2012) address this issue by linking market data on credit default swaps with digital put options and translate the observed premium into an implied volatility using a Black-Scholes approach. The reported implied volatilities for a CoCo issued by Llyods Banking Group ranged between 77 and 114\%, which seems high even for deep-out-of-the-money options. Thus, we followed a more pragmatic course of action and treated volatility as a constant parameter equal to 50\%. More sophisticated approaches such as implicit estimates did neither improve the models accuracy nor result in a different relation between the models.

We fit the model at one single calibration date $t-1$ via the trigger barrier $\underline{S}$ to the market price $P_{t-1}$ using all observable information, e.g., in case of the extended model $\Omega_{t-1}=\left\{S_{t-1}, c s_{t-1}, r_{t-1}, q_{t-1}\right\}$. The trigger barrier that explains the market price is our estimate $\underline{\widehat{S}}$ for the unobservable trigger barrier at the pricing date $t$. While Wilkens and Bethke (2014) only calibrate the model at the issue date and keep the implicit barrier fixed for all subsequent pricing dates, we repeat the calibration for each pricing date of our sample. This enables us to get more recent market sentiment as well as the regulatory trigger which is not necessarily constant over time.

For given conversion terms, the pricing function of the theoretical models is $\mathrm{u}$ shaped in the trigger barrier that results in situations in which two barriers satisfy the calibration condition. This arises from the fact that a lower barrier makes a trigger 


\section{Calibration at $t-1$ :}

Calibration of model $F(\Omega, \Theta)$ to market prices $P$ via $\Theta$ over estimation window of $\mathrm{s}$ days

$$
\widehat{\Theta}=\underset{\beta_{S}, \beta_{c s}, \beta_{r}}{\operatorname{argmin}} \sum_{i=t-s}^{t-1}\left[P_{i}-F\left(\Omega_{i}, \beta_{S}, \beta_{c s}, \beta_{r}\right)\right]^{2}
$$$$
\underbrace{t-1}_{\begin{array}{c}
\text { Estimation window } \begin{array}{c}
\text { of } s \text { days } \\
\text { of }
\end{array} \\
\begin{array}{c}
\text { Calibration } \\
\text { date }
\end{array}
\end{array}}
$$

Fig. 3 Time structure of market-based model
Pricing at $t$ :

Comparison of model price $F\left(\Omega_{t}, \widehat{\Theta}\right)$ and market price $P_{t}$

$$
F\left(\Omega_{t}, \widehat{\Theta}\right)-P_{t}
$$

event, usually associated with a loss, less likely and therefore leads to a higher CoCo value. Conversely, a higher barrier can increase the CoCo value too, since it makes the share package received upon conversion more valuable. In line with De Spiegeleer and Schoutens (2011), we use the lower barrier in these cases. Of course, for write-down CoCos the pricing function is monotonically decreasing in the barrier since the loss inevitably associated with the trigger event becomes more likely.

In case of the market-based trading model, we follow a different procedure which is illustrated in Fig. 3. We estimate the risk exposures $\beta_{S}, \beta_{c s}, \beta_{r}$ of the market-based model by using historical data out of an estimation window of $s=100$ trading days that ends at the calibration date at $t-1$. Using time series regression, we get the set of estimates $\widehat{\Theta}=\left\{\widehat{\beta}_{S}, \widehat{\beta}_{c s}, \widehat{\beta}_{r}\right\}$ which are used to price the CoCo out-of-sample at $t$ with the model $F\left(\Omega_{t}, \widehat{\Theta}\right)$.

For each model, we compute the relative pricing error $R P E_{t}$ at the pricing date $t$, which is given by the deviation of the model price $F\left(\Omega_{t}, \widehat{\Theta}\right)$ from the realized market price $P_{t}$ divided by the market price $P_{t}$ :

$$
R P E_{t}=\frac{F\left(\Omega_{t}, \widehat{\Theta}\right)-P_{t}}{P_{t}}
$$

\subsection{Model comparison}

In order to assess the out-of-sample pricing performance of the regarded models, we calculate different error metrics to identify the strengths and weaknesses of the individual models. First, we focus on the sample average of the considered metrics. The results are presented in Table 2.

In principal, an appropriate pricing model should on average capture the realized market price, i.e., it should be unbiased. The mean relative error (ME) of all models is between -0.030 to -0.003 percentage points (pts). Since the CoCo trades on average around par, an error of about -3 cents is rather negligible. This indicates a proper pricing performance of all models. Yet, there is a slight advantage of the extended model (EM) and the trading model (TM) over the standard model (SM) and the default risk model (DM). 
Table 2 Pricing performance, sample average

\begin{tabular}{lllll}
\hline Metric & Theory-based & & Market-based \\
\cline { 2 - 4 } & SM & DM & EM & TM \\
\hline ME & -0.030 & -0.020 & -0.011 & -0.003 \\
EV & 0.930 & 0.821 & 0.663 & 0.604 \\
RMSE & 0.945 & 0.837 & 0.663 & 0.604 \\
QE & 4.513 & 3.967 & 2.936 & 3.006 \\
\hline
\end{tabular}

This table shows the error metrics in percentage points (pts) for the four pricing models. All metrics are based on the relative pricing error (RPE). 'Mean Error (ME)' is the average of the RPE. 'Error Variation $(\mathrm{EV})$ ' is the standard deviation of the RPE. 'Root Mean Squared Error (RMSE)' is the square root of average squared RPE. 'Quantile Error (QE)' is the 99\%-quantile of the absolute RPE. SM represents the standard model, DM stands for the default risk model, EM denotes the extended model and TM is the market-based trading model. We used 100.000 paths and weekly steps in our simulation algorithm for the extended model

Besides a low level of errors, a more stable price prediction is desirable. This characteristic can be measured in terms of error variation (EV) which we calculate as the standard deviation of the relative pricing error. Comparing the error variation of the SM with the DM, we observe a lower error variation using the DM, which shows that information on the issuer's solvency stabilizes the price prediction. In addition, the EM reduces the error variation of the DM by 0.158 pts or $19 \%$, proving that consideration of the effective maturity structure (in the EM) further stabilizes the price prediction. Remarkably, the trading model without any pricing theoretical considerations produces the most stable results.

Taking bias and stability into account, we obtain a clear preference order across all models. Within the theory-based models, the SM with its simplified structure produces reasonable price predictions. However, the DM dominates the SM with respect to both stability and accuracy. This leads to the conclusion that information on credit risk is crucial to price CoCos accurately and must therefore be considered in a pricing model.

\section{Summary (The Effect of Credit Risk on Pricing Accuracy)}

Augmenting the models with information on the issuer's solvency stabilizes the pricing performance. Therefore, information on credit risk is essential for accurate CoCo pricing and should consequently be included in a pricing model.

Although mean error and error variation are suitable metrics for assessing systematic pricing bias and stability, one metric which reflects both simultaneously is desirable. In this study we calculate the average root mean squared relative error (RMSE) of all CoCos in our sample. This metric accounts for the fact that a model with a given average accuracy is penalized for a higher error variation.

The root mean squared error confirms the previously discussed preference order. The sophisticated EM shows the best pricing performance of the theory-based models with a root mean squared error of 0.663 pts, while the DM has a root mean squared error of 0.821 pts. The DM is more accurate than the SM which has a root mean squared error of 0.945 pts. On average, the TM resulted in the most accurate pricing performance with a root mean squared error of 0.604 pts. 
Table 3 t-values for mean difference between forecast errors of corresponding model combinations

\begin{tabular}{lllll}
\hline & SM & DM & EM & TM \\
\hline SM & - & 3.673 & 8.808 & 10.161 \\
DM & - & - & 8.605 & 10.396 \\
EM & - & - & - & 6.474 \\
TM & - & - & - & - \\
\hline
\end{tabular}

This table shows the 't-values' for the mean difference of the daily absolute forecast errors between each combination of two models for the whole CoCo sample and the sample period

While the SM and the DM are based on the same restrictive maturity assumptions, the EM regards the perpetual lifetime, the initial blocking period as well as the issuer's call decision. By comparing the DM with the EM, we can assess the effect of the maturity assumptions on the pricing performance, given the model already regards the issuer's solvency. We observe that a flexible maturity structure results in a 0.174 pts or $21 \%$ lower root mean squared error of the EM compared to the DM and thus leads to a more accurate and stable price prediction. Accordingly, we conjecture that correctly modelling the effective lifetime improves the predictive pricing accuracy of AT1 CoCos considerably. Thus, increasing a model's complexity to include credit risk and allow for a more realistic maturity structure is not only justified, but necessary for accurate CoCo pricing.

\section{Summary (The Effect of Maturity Structure on Pricing Accuracy)}

Since CoCos are perpetual and callable by the issuer after an initial blocking period, accurate pricing requires a model that flexibly accounts for the product's effective lifetime.

The TM still yields the most accurate pricing performance on average. At first glance, this result seems surprising since the TM disregards the CoCo's product characteristics and focuses solely on the risk factors it is exposed to. However, in contrast to the calibration procedure of the theory-based models, for which the trigger barrier estimate is extracted at one single date, the empirical risk exposures of the TM are estimated across a period of $s$ days. The finding that the TM on average outperforms the theorybased models indicates that higher model complexity is not always necessary and that a simple model only using historic market data without pricing theory can at times be sufficient.

Table 3 contains the t-values for the mean of absolute forecast errors according to model A (displayed in rows) subtracted by the absolute forecast errors from model B (displayed in the corresponding column). For all model combinations, we find a significant effect in the sense that mean forecasts errors from a given model are statistically different from those of another model.

For the sake of completeness, we also conducted large pricing error investigations by computing the quantile error (QE) as the $99 \%$-quantile of the absolute relative pricing error to assess whether a particular model results in severe mispricing. In fact, this results in a slightly different preference order. The $99 \%$-quantile suggests that the EM with a quantile error of 2.936 pts is less prone to severe mispricing than the TM with a quantile error of 3.004 pts. A reason for this superior performance of the EM 
relative to the TM in case of extreme errors might be the ability of the EM to capture the non-linear CoCo sensitivities towards the risk factors which cannot be accomplished by the TM. For large price changes these non-linearities can be significant. For the optimal model choice, both the average pricing accuracy and the risk of landing in the tails of the error distribution must be considered.

\section{Summary (Trade-off: Accuracy vs. Error Tail-Risk)}

On average, the trading model which disregards the product characteristics and is based solely on historic market data outperforms the theory-based models in terms of root mean squared error. However, consideration of the 99\%-quantile of the pricing errors reveals that a more complex model with a theoretical foundation is required to prevent severe mispricing. Thus, the decision which model to apply in practice entails a trade-off between average pricing accuracy and the risk of severe mispricing.

To provide a deeper understanding of our results, we report the root mean squared error on an individual CoCo level for the considered models in Table 4. The root mean squared errors for all CoCos and models are in the range between 0.337 pts and 2.232 pts. This underlines the result that CoCos can be priced quite accurately. Furthermore, there is a clear preference order within the theory-based models: Consideration of the credit risk significantly improves the accuracy and in total the EM is significantly more precise than the DM which indicates that the effective maturity structure matters.

\section{Summary (Accurate Pricing Performance of Individual CoCos)}

While the EM and TM show a superior pricing performance relative to SM and DM, all models are able to price CoCos properly. Hence, accurate CoCo pricing is not only possible in the aggregate but also on an individual CoCo level.

The only exceptions of this order are BBVA (2) for which the EM is outperformed by the SM and the DM and HSBC (2) for which the DM outperforms the EM. Comparing the individual pricing performance of EM with TM, we observe that the EM has priced the two issues of Banco Popular Español and the issue of Allied Irish Banks more accurately than the TM. A closer look reveals that these CoCos share the similarity of being part of the class of CoCos for which all four models showed the least accurate pricing results. An explanation for this observation might be the fact that both issuing banks experienced severe trouble within our sample period. This is in line with the observed quantile errors in Table 2 which suggest a superior pricing performance of the EM over the TM once non-linear CoCo price dynamics emerge. The EM is considered as being the more robust model in distress.

\section{Performance in distress}

This section focuses on distressed situations of the CoCo during which a trigger event becomes closer and the CoCo changes its nature. Figure 4 contains the price evolution of the CoCo Ba. Popular (1). During sideways market phases, the CoCo trades relatively stable at the same level similar to a coupon bond. However, once the CoCo value drops, a strong increase in volatility can be observed and an equity-like 
Table 4 Root mean squared error, individual level

\begin{tabular}{|c|c|c|c|c|}
\hline \multirow[t]{2}{*}{ CoCo } & \multicolumn{3}{|c|}{ Theory-based } & \multirow{2}{*}{$\begin{array}{l}\text { Market-based } \\
\text { TM }\end{array}$} \\
\hline & SM & DM & EM & \\
\hline Aareal Bank & 1.214 & - & 0.925 & 0.821 \\
\hline ABN AMRO & 0.601 & 0.587 & 0.542 & 0.514 \\
\hline Allied Irish Banks & 1.974 & 1.750 & 1.270 & 1.272 \\
\hline BBVA (1) & 0.621 & 0.606 & 0.602 & 0.573 \\
\hline BBVA (2) & 0.541 & 0.556 & 0.565 & 0.521 \\
\hline BBVA (3) & 0.506 & 0.468 & 0.428 & 0.411 \\
\hline Ba. Popular (1) & 1.960 & 1.378 & 0.887 & 0.924 \\
\hline Ba. Popular (2) & 2.232 & 1.847 & 1.166 & 1.304 \\
\hline Bank of Ireland & 1.032 & 0.868 & 0.692 & 0.616 \\
\hline Barclays (1) & 0.746 & 0.702 & 0.598 & 0.536 \\
\hline Barclays (2) & 0.760 & 0.714 & 0.576 & 0.505 \\
\hline BNP Paribas & 0.508 & 0.478 & 0.445 & 0.381 \\
\hline Crédit Agricole & 0.592 & 0.558 & 0.517 & 0.472 \\
\hline Danske Bank (1) & 0.750 & 0.730 & 0.476 & 0.367 \\
\hline Danske Bank (2) & 0.534 & 0.494 & 0.470 & 0.337 \\
\hline Deutsche Bank & 1.050 & 0.936 & 0.777 & 0.685 \\
\hline HSBC (1) & 0.841 & 0.829 & 0.586 & 0.451 \\
\hline HSBC (2) & 0.588 & 0.559 & 0.570 & 0.496 \\
\hline Intesa Sanpaolo & 0.837 & 0.767 & 0.646 & 0.628 \\
\hline KBC Group & 0.613 & 0.578 & 0.537 & 0.471 \\
\hline Lloyds Banking & 0.609 & 0.561 & 0.488 & 0.416 \\
\hline Permanent TSB & 1.504 & 1.273 & 0.938 & 0.753 \\
\hline Santander (1) & 0.794 & 0.724 & 0.643 & 0.592 \\
\hline Santander (2) & 0.817 & 0.734 & 0.690 & 0.605 \\
\hline Société Générale & 1.099 & 1.065 & 0.477 & 0.422 \\
\hline UBS & 0.558 & 0.525 & 0.496 & 0.389 \\
\hline UniCredit & 1.643 & 1.470 & 0.896 & 0.862 \\
\hline Average & 0.945 & 0.837 & 0.663 & 0.604 \\
\hline
\end{tabular}

This table shows the relative 'Root Mean Squared Error (RMSE)' of daily predictions of all four models for the whole CoCo sample on an individual product level. SM represents the standard model, DM stands for the default risk model, EM denotes the extended model and TM is the market-based trading model. We used 100.000 paths and weekly steps in our simulation algorithm for the extended model

character becomes visible. This observation highlights the challenge of understanding CoCos in a distressed situation because their product characteristics might change.

We define distress as a state in which the CoCo return exceeds twice its historical volatility. We then calculate our error metrics based only on dates during which the CoCo was in a distressed state. Table 5 shows the resulting conditional error metrics. As expected, the pricing accuracy of each model suffers when it comes to distress. This is reflected by the more pronounced conditional error metrics shown in comparison to 


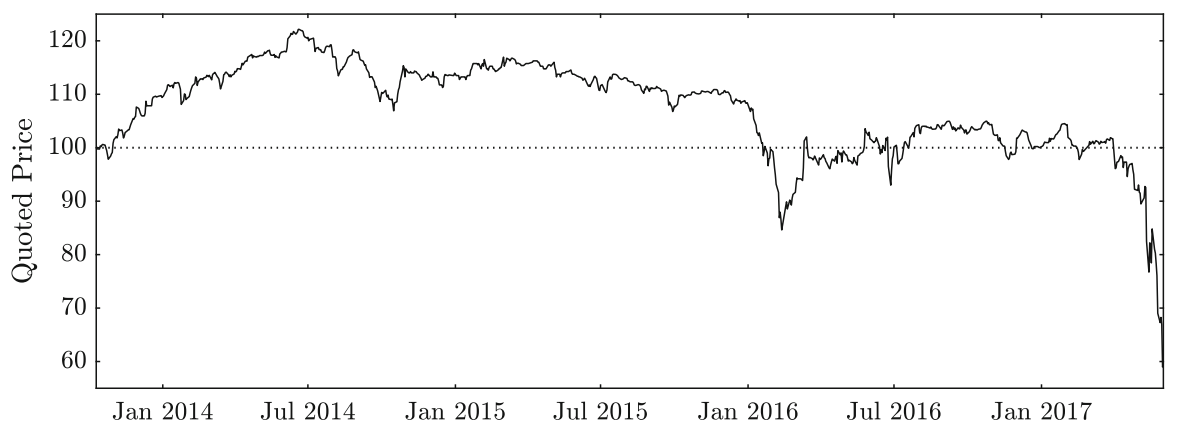

Fig. 4 Exemplary price evolution of CoCo Ba. Popular (1).

This figure shows the evolution of the quoted (clean) price of the CoCo Ba. Popular (1) issued by Banco Popular Español on October 10, 2013. The dotted line indicates the par value. The CoCo was triggered by the ECB on June 7, 2017

Table 5 Pricing performance in distress, sample average

\begin{tabular}{|c|c|c|c|c|}
\hline \multirow[t]{2}{*}{ Metric } & \multicolumn{3}{|c|}{ Theory-based } & \multirow{2}{*}{$\begin{array}{l}\text { Market-basec } \\
\text { TM }\end{array}$} \\
\hline & SM & DM & EM & \\
\hline ME & -0.162 & -0.129 & -0.070 & -0.044 \\
\hline $\mathrm{EV}$ & 1.698 & 1.545 & 1.283 & 1.326 \\
\hline RMSE & 1.712 & 1.559 & 1.294 & 1.357 \\
\hline
\end{tabular}

This table shows the conditional error metrics in percentage points (pts) for the four pricing models when the CoCo is in distress. We define distress as a CoCo return that exceeds two standard deviations of the historical CoCo return. All metrics are based on the relative pricing error (RPE). 'Mean Error (ME)' is the average of the RPE. 'Error Variation (EV)' is the standard deviation of the RPE. 'Root Mean Squared Error (RMSE)' is the square root of average squared RPE. SM represents the standard model, DM stands for the default risk model, EM denotes the extended model and TM is the market-based trading model. We used 100.000 paths and weekly steps in our simulation algorithm for the extended model

the results on average reported in Table 2 . With a mean error between -0.162 pts to -0.044 pts the models reveal a moderate but clearly observable underpricing in times of distress when compared to the almost non-existing ME for the total sample period.

Regarding stability, we observe the expected picture of a higher error variation for all the models. However, in contrast to error variation for the total period, we observe that in distress the EM clearly shows a more stable price prediction than the TM. This finding is confirmed by the result of the conditional root mean squared errors. With a root mean squared error of 1.294 pts the EM shows indeed the most stable and accurate pricing performance of all the models and now even outperforms the TM with a conditional root mean squared error of 1.357 pts. The other theory-based models, SM and DM, are also better in their relative performance in terms of the root mean squared error. We therefore conclude that in distressed situations, in which a trigger event becomes more likely, a simple trading approach is comparably more prone to severe mispricing than a sophisticated theory model like the EM. This is reflected in a high error variation resulting in a comparably high conditional root mean squared error. In contrast, a theory-based model that accounts for the individual characteristics 
Table 6 t-values for mean difference between forecast errors of corresponding model combinations in distress

\begin{tabular}{lllll}
\hline & SM & DM & EM & TM \\
\hline SM & - & 1.537 & 7.991 & 7.604 \\
DM & - & - & 7.681 & 8.051 \\
EM & - & - & - & 2.754 \\
TM & - & - & - & - \\
\hline
\end{tabular}

This table shows the 't-values' for the mean difference of the daily absolute forecast errors between each combination of two models for the whole CoCo sample in distressed states

of a CoCo produces a robust price prediction. Apparently, when in distress, the nonlinear sensitivities towards the risk factors of the EM better capture the changed CoCo characteristics than the linear sensitivities of the TM.

\section{Summary (Pricing Performance in Distress)}

In distress, a robust price prediction requires a sophisticated model with a theoretical foundation that captures the non-linear sensitivities towards risk factors.

Table 6 contains the t-values for the mean difference of the absolute forecast errors between the models in distressed states. In line with Table 3, we find statistically significant differences at a $1 \%$ level except for one particular combination between $\mathrm{SM}$ and DM. The corresponding t-value in this case equals 1.537 and corresponds to a still reasonable p-value of 0.061 .

Table 7 shows the conditional root mean squared error on an individual CoCo basis. It can be seen that the conditional root mean squared errors of all CoCos are more pronounced compared to the results from total period which are reported in Table 4. In distress there are nine cases in which the EM shows a higher accuracy than the TM, compared to three cases for the total period. Furthermore, while the TM strictly outperforms the DM for all CoCos during the total period, in distress the DM shows a lower conditional root mean squared error in five cases. This relative catch-up effect of the theory-based models compared to the market-based model confirms that it is necessary to consider the CoCo's product characteristics in a model in order to obtain an accurate price prediction in distress.

Focusing on the theory-based models, we also recognize that the SM and the DM come closer in precision to the EM. Compared to the EM, the SM and DM showed lower root mean squared error in four and five cases respectively. This finding indicates that in distressed states, the importance of the call decision and the perpetual lifetime become comparably less important than the changing sensitivities associated with a likely trigger event. As expected, comparing the DM with the SM we still observe a clear improved pricing accuracy when information on the issuers solvency is included. Relative to the root mean squared error improvement for the total sample period of 0.108 pts, the inclusion of credit risk leads to a reduction of 0.153 pts in distress.

Combining the result from within the class of theory-based models with the result across model classes, we conclude that especially in distress the CoCo's sensitivity with respect to the more likely trigger event is important which cannot be captured 
Table 7 Root mean squared error in distress, individual level

\begin{tabular}{lllll}
\hline CoCo & \multicolumn{2}{l}{ Theory-based } & & Market-based \\
\cline { 2 - 4 } & SM & DM & EM & TM \\
\hline Aareal Bank & 2.506 & - & 2.193 & 2.355 \\
ABN AMRO & 1.650 & 1.400 & 1.054 & 0.996 \\
Allied Irish Banks & 3.571 & 3.290 & 2.609 & 3.440 \\
BBVA (1) & 1.247 & 1.207 & 1.241 & 1.150 \\
BBVA (2) & 1.256 & 1.276 & 1.385 & 1.224 \\
BBVA (3) & 0.787 & 0.755 & 0.789 & 0.780 \\
Ba. Popular (1) & 3.184 & 3.027 & 1.163 & 1.345 \\
Ba. Popular (2) & 4.504 & 4.040 & 2.450 & 3.973 \\
Bank of Ireland & 2.297 & 1.921 & 1.297 & 1.120 \\
Barclays (1) & 1.701 & 1.628 & 1.303 & 1.234 \\
Barclays (2) & 1.544 & 1.471 & 1.126 & 1.099 \\
BNP Paribas & 0.998 & 0.956 & 0.940 & 0.908 \\
Crédit Agricole & 1.009 & 0.969 & 0.918 & 0.936 \\
Danske Bank (1) & 1.153 & 1.085 & 0.836 & 0.773 \\
Danske Bank (2) & 1.051 & 0.942 & 0.823 & 0.747 \\
Deutsche Bank & 2.220 & 2.024 & 1.964 & 1.993 \\
HSBC (1) & 1.149 & 1.088 & 1.207 & 1.115 \\
HSBC (2) & 1.326 & 1.270 & 1.348 & 1.304 \\
Intesa Sanpaolo & 1.162 & 1.111 & 0.858 & 0.849 \\
KBC Group & 1.219 & 1.198 & 1.179 & 1.119 \\
Lloyds Banking & 1.326 & 1.258 & 1.016 & 0.910 \\
Permanent TSB & 1.989 & 1.674 & 1.252 & 1.122 \\
Santander (1) & 1.466 & 1.346 & 1.272 & 1.294 \\
Santander (2) & 1.475 & 1.323 & 1.308 & 1.364 \\
Société Générale & 1.367 & 1.294 & 0.704 & 0.700 \\
UBS & 1.100 & 1.033 & 1.030 & 0.908 \\
UniCredit & 2.291 & 2.178 & 1.822 & 1.952 \\
Average & $\mathbf{1 . 7 1 2}$ & $\mathbf{1 . 5 5 9}$ & $\mathbf{1 . 2 9 4}$ & $\mathbf{1 . 3 5 7}$ \\
\hline This tableshown & & & \\
\hline
\end{tabular}

This table shows the conditional relative 'Root Mean Squared Error (RMSE)' of all four models sample on an individual product level. We condition on the pricing dates at which the CoCo is in a state of distress. SM represents the standard model, DM stands for the default risk model, EM denotes the extended model and TM is the market-based trading model. We used 100.000 paths and weekly steps in our simulation algorithm for the extended model

by the market-based model. Hence, accurate CoCo pricing in distress requires more complex theory-based models.

In total, based on the results, we conclude that especially in distress the CoCo's sensitivity with respect to the increased likelihood of a trigger event is important. As this cannot be captured by the market-based model, accurate CoCo pricing in distress requires more complex theory-based models. 


\section{Conclusion}

This study provides an analysis of CoCo pricing models. For this reason, we considered four different pricing models and assessed their out-of-sample pricing ability using a broad dataset of 27 CoCos which comprises all issuances between January 1, 2013 and May 31, 2016 denominated in euros.

We regarded four models: a standard model proposed by De Spiegeleer and Schoutens (2012) which relies on a highly restrictive maturity assumption in order to obtain closed-form pricing formulae. A modified version of the standard model with which we allow for a sudden default of the issuer to incorporate credit risk but still impose restrictive maturity assumptions for the sake of a closed form solution. An extended model that flexibly accounts for the CoCos corresponding lifetime by regarding the effective trigger date, the perpetual lifetime as well as the issuer call right after an initial blocking period. A market-based model without any pricing-theoretical background that solely relies on historical data.

To the best of our knowledge this is the first serious empirical study in the literature on CoCos dedicated to assess the out-of-sample pricing performance of different models. Our study reveals four major results: First, information on the issuer's solvency stabilizes the pricing performance and must therefore be included in a CoCo pricing model. Second, capturing the effective lifetime of the CoCo by incorporating more product characteristics into the model further improves the out-of-sample accuracy of theory-based models. Thus, within the class of theory-based models, increasing the complexity improves pricing accuracy. Third, in a normal market environment, on average a more sophisticated theory-based model is not advantageous over a marketbased trading model. Last, once the CoCo turns into distress, however, the trading model is comparably more exposed to severe mispricing than a more complex theorybased model. In this case the more sophisticated EM outperforms the market-based approach.

From these findings we conclude that in a normal market environment a simple model only based on historic data can be sufficient for the pricing of CoCos. In this case increasing a model's complexity does not necessarily improve its out-of-sample pricing ability. However, in distress, the simple trading model leads to severe mispricing since it cannot capture the changing (non-linear) CoCo sensitivities once the trigger event becomes more likely. Therefore, distressed states require a sophisticated model with a theoretical foundation in order to price CoCos accurately.

Funding Open Access funding enabled and organized by Projekt DEAL.

Open Access This article is licensed under a Creative Commons Attribution 4.0 International License, which permits use, sharing, adaptation, distribution and reproduction in any medium or format, as long as you give appropriate credit to the original author(s) and the source, provide a link to the Creative Commons licence, and indicate if changes were made. The images or other third party material in this article are included in the article's Creative Commons licence, unless indicated otherwise in a credit line to the material. If material is not included in the article's Creative Commons licence and your intended use is not permitted by statutory regulation or exceeds the permitted use, you will need to obtain permission directly from the copyright holder. To view a copy of this licence, visit http://creativecommons.org/licenses/by/4.0/. 


\section{Appendix}

\section{Derivation of state prices}

A state price with perpetual lifetime written on the stock price $S$ follows the following ordinary differential equation (ODE):

$$
\frac{1}{2} \sigma^{2} S_{t}^{2} \frac{\partial^{2} P}{\partial S^{2}}+(r-q+\lambda) S_{t} \frac{\partial P}{\partial S}-(r+\lambda) P_{t}=0
$$

We can obtain a closed-form solution for a state price which satisfies the ODE in Eq. (19) by using the general relationship

$$
C_{t}=c_{1} S_{t}^{\gamma_{1}}+c_{2} S_{t}^{\gamma_{2}}
$$

in which $c_{1}$ and $c_{2}$ are arbitrary constants and the exponents $\gamma_{1}$ and $\gamma_{2}$ are given by:

$$
\gamma_{1}=\frac{1}{\sigma^{2}}\left[q-r-\lambda+\frac{1}{2} \sigma^{2}-\sqrt{2(r+\lambda) \sigma^{2}+\frac{1}{4}\left(2(q-r-\lambda)+\sigma^{2}\right)^{2}}\right]
$$

and

$$
\gamma_{2}=\frac{1}{\sigma^{2}}\left[q-r-\lambda+\frac{1}{2} \sigma^{2}+\sqrt{2(r+\lambda) \sigma^{2}+\frac{1}{4}\left(2(q-r-\lambda)+\sigma^{2}\right)^{2}}\right]
$$

Using the barriers $\underline{S}$ and $\bar{S}$ as boundary conditions, we derive the value of a state price $\underline{C}_{t}$ for the trigger event and that for the issuer call $\bar{C}_{t}$.

The boundary conditions for the trigger state price $\underline{C}_{t}$ are:

$$
\underline{C}_{t}=\left\{\begin{array}{l}
1, S_{t} \rightarrow \underline{S} \\
0, S_{t} \rightarrow \bar{S}
\end{array}\right.
$$

Using this boundary condition in Eq. (21), we can determine the constants $c_{1}$ and $c_{2}$ in Eq. (20) and obtain the trigger state price $\underline{C}_{t}$

$$
\underline{C}_{t}=\frac{1}{\underline{S}^{\gamma_{1}}-\underline{S}^{\gamma_{2}} \bar{S}^{\gamma_{1}-\gamma_{2}}} S^{\gamma_{1}}+\frac{1}{\underline{S}^{\gamma_{2}}-\underline{S}^{\gamma_{1}} \bar{S}^{\gamma_{2}-\gamma_{1}}} S^{\gamma_{2}}
$$

Similarly, the boundary conditions for the call state price $\bar{C}_{t}$ read:

$$
\bar{C}_{t}=\left\{\begin{array}{l}
0, S_{t} \rightarrow \frac{S}{\bar{S}} \\
1, S_{t} \rightarrow \bar{s}
\end{array}\right.
$$


Solving for the constants $c_{1}$ and $c_{2}$ in Eq. (20), we get the call state price $\bar{C}_{t}$ :

$$
\bar{C}_{t}=\frac{1}{\bar{S}^{\gamma_{1}}-\bar{S}^{\gamma_{1}} \underline{S}^{\gamma_{1}-\gamma_{2}}} S^{\gamma_{1}}+\frac{1}{\bar{S}^{\gamma_{2}}-\bar{S}^{\gamma_{1}} \underline{S}^{\gamma_{2}-\gamma_{1}}} S^{\gamma_{2}}
$$

\section{References}

Altman, E., Brady, B., Resti, A., \& Sironi, A. (2005). The link between default and recovery rates: Theory, empirical evidence, and implications. The Journal of Business, 78, 2203-2228.

Avdjiev, S., Bogdanova, B., Bolton, P., Jiang, W., \& Kartasheva, A. (2017). Coco issuance and bank fragility. BIS working papers 678 .

Black, F., \& Scholes, M. (1973). The pricing of options and corporate liabilities. Journal of Political Economy, 81, 637-654.

Brennan, M., \& Schwartz, E. S. (1977). Convertible bonds: Valuation and optimal strategies for call and conversion. Journal of Finance, 32.

Brigo, D., Garcia, J., \& Pede, N. (2015). Coco bonds pricing with credit and equity calibrated first-passage firm value models. International Journal of Theoretical and Applied Finance, 18, 1550015.

Buergi, M. (2013). Pricing contingent convertibles: A general framework for application in practice. Financial Markets and Portfolio Management, 27, 31-63.

Cheridito, P., \& Xu, Z. (2015). A reduced-form contingent convertible bond model with deterministic conversion intensity. Journal of Risk, 17, 1-18.

Corcuera, J., De Spiegeleer, J., Ferreiro-Castilla, A., Kyprianou, A. E., Madan, D., \& Schoutens, W. (2013). Pricing of contingent convertibles under smile conform models. The Journal of Credit Risk, 9, 121-140.

De Spiegeleer, J., \& Schoutens, W. (2012). Pricing contingent convertibles: A derivatives approach. Journal of Derivatives, 20, 27-36.

De Spiegeleer, J., \& Schoutens, W. (2011). Pricing contingent convertibles: A derivatives approach, working Paper.

De Spiegeleer, J., \& Schoutens, W. (2014). Coco bonds with extension risk. Wilmott Magazine, 2014, 78-91.

Flannery, M. J. (2016). Stabilizing large financial institutions with contingent capital certificates. Quarterly Journal of Finance, 6.

Ingersoll, J. (1977). A contingent-claims valuation of convertible securities. Journal of Financial Economics, 4, 289-321.

Pennacchi, G. (2011). A structural model of contingent bank capital, nBER Working Paper.

Pennacchi, G., Tchistyi, A. (2018). Contingent convertibles with stock price triggers: The case of perpetuities. The Review of Financial Studies Forthcoming.

Rubinstein, M., \& Reiner, E. (1991a). Breaking down the barriers. RISK, 4, 28-35.

Rubinstein, M., \& Reiner, E. (1991b). Unscrambling the binary code. Risk Magazine, 4, 75-83.

Sarkar, S. (2003). Early and late calls of convertible bonds: Theory and evidence. Journal of Banking \& Finance, 27, 1349-1374.

Serjantov, A. (2011). On practical pricing hybrid capital securities. In: Presentation, global derivatives trading and risk management.

Svensson, L. E. (1994). Estimating and interpreting forward interest rates: Sweden 1992-1994, working Paper.

Turfus, C., \& Shubert, A. (2017). Analytic pricing of coco bonds. International Journal of Theoretical and Applied Finance, 20, 1750034.

Wilkens, S., \& Bethke, N. (2014). Contingent convertible (coco) bonds: A first empirical assessment of selected pricing models. Financial Analysts Journal, 70, 59-77.

Publisher's Note Springer Nature remains neutral with regard to jurisdictional claims in published maps and institutional affiliations. 